\title{
ON THE ACTION OF LIPSCHITZ FUNCTIONS ON VECTOR-VALUED RANDOM SUMS
}

\author{
JAN VAN NEERVEN AND MARK VERAAR
}

\begin{abstract}
Let $X$ be a Banach space and let $\left(\xi_{j}\right)_{j} \geqslant 1$ be an i.i.d. sequence of symmetric random variables with finite moments of all orders. We prove that the following assertions are equivalent:
\end{abstract}

(1) There exists a constant $K$ such that

$$
\left(\mathbb{E}\left\|\sum_{j=1}^{n} \xi_{j} f\left(x_{j}\right)\right\|^{2}\right)^{\frac{1}{2}} \leqslant K\|f\|_{\operatorname{Lip}}\left(\mathbb{E}\left\|\sum_{j=1}^{n} \xi_{j} x_{j}\right\|^{2}\right)^{\frac{1}{2}}
$$

for all Lipschitz functions $f: X \rightarrow X$ satisfying $f(0)=0$ and all finite sequences $x_{1}, \ldots, x_{n}$ in $X$.

(2) $X$ is isomorphic to a Hilbert space.

For Banach spaces $X$ and $Y$ let $\operatorname{Lip}_{0}(X, Y)$ denote the Banach space of all Lipschitz continuous functions $f: X \rightarrow Y$ satisfying $f(0)=0$ with norm $\|f\|_{\text {Lip }}:=$ $L_{f}$, the Lipschitz constant of $f$. Our main result relates the action of functions $f \in \operatorname{Lip}_{0}(X, Y)$ on random sums in $X$ with the cotype and type of $X$ and $Y$, respectively. Since the best constants are obtained for Gaussian variables, we state the result for this case first.

Theorem 1. Let $X$ and $Y$ be Banach spaces with $\operatorname{dim} X=\infty$ and $\operatorname{dim} Y \geqslant 1$, and let $\left(\gamma_{j}\right)_{j \geqslant 1}$ be a sequence of independent standard Gaussian random variables. The following assertions are equivalent:

(i) For all finite sequences $x_{1}, \ldots, x_{n} \in X$, all scalars $a_{1}, \ldots, a_{n}>0$, and all $f_{1}, \ldots, f_{n} \in \operatorname{Lip}_{0}(X, Y)$ we have

$$
\left(\mathbb{E}\left\|\sum_{j=1}^{n} \gamma_{j} a_{j}^{-1} f_{j}\left(a_{j} x_{j}\right)\right\|^{2}\right)^{\frac{1}{2}} \leqslant K\left(\max _{1 \leqslant j \leqslant n}\left\|f_{j}\right\|_{\text {Lip }}\right)\left(\mathbb{E}\left\|\sum_{j=1}^{n} \gamma_{j} x_{j}\right\|^{2}\right)^{\frac{1}{2}}
$$

where $K$ is a constant depending on $X$ and $Y$ only.

(ii) For all finite sequences $x_{1}, \ldots, x_{n} \in X$ there exist scalars $a_{1}, \ldots, a_{n}>0$ such that for all $f \in \operatorname{Lip}_{0}(X, Y)$ we have

$$
\left(\mathbb{E}\left\|\sum_{j=1}^{n} \gamma_{j} a_{j}^{-1} f\left(a_{j} x_{j}\right)\right\|^{2}\right)^{\frac{1}{2}} \leqslant K\|f\|_{\text {Lip }}\left(\mathbb{E}\left\|\sum_{j=1}^{n} \gamma_{j} x_{j}\right\|^{2}\right)^{\frac{1}{2}},
$$

where $K$ is a constant depending on $X$ and $Y$ only.

Date: April 22, 2005.

2000 Mathematics Subject Classification. Primary: 46C15, Secondary: 46B09, 47B10.

Key words and phrases. Lipschitz functions, type 2, cotype 2, isomorphic characterization of Hilbert spaces, Dvoretzky's theorem.

The authors are supported by the 'VIDI subsidie' 639.032.201 in the 'Vernieuwingsimpuls' programme of the Netherlands Organization for Scientific Research (NWO) and by the Research Training Network HPRN-CT-2002-00281. 
(iii) $X$ has cotype 2 and $Y$ has type 2 .

If (i) or (ii) holds with constant $K$, then the Gaussian cotype 2 constant of $X$ and the Gaussian type 2 constant of $Y$ satisfy $C_{2}^{\gamma}(X) \leqslant K$ and $T_{2}^{\gamma}(Y) \leqslant \sqrt{2} K$.

Proof. The implication (i) $\Rightarrow$ (ii) is trivial.

(ii) $\Rightarrow$ (iii): First we prove that $X$ has cotype 2 with $C_{2}^{\gamma}(X) \leqslant K$. Fix a norm one vector $y_{0} \in Y$ and define $f \in \operatorname{Lip}_{0}(X, Y)$ by $f(x):=\|x\| y_{0}$. Since $\|f\|_{\text {Lip }}=1$ it follows that for $x_{1}, \ldots, x_{n} \in X$ we have, with the $a_{1}, \ldots, a_{n}>0$ as in (ii),

$$
\sum_{j=1}^{n}\left\|x_{j}\right\|^{2}=\mathbb{E}\left\|\sum_{j=1}^{n} \gamma_{j} f\left(x_{j}\right)\right\|^{2}=\mathbb{E}\left\|\sum_{j=1}^{n} \gamma_{j} a_{j}^{-1} f\left(a_{j} x_{j}\right)\right\|^{2} \leqslant K^{2} \mathbb{E}\left\|\sum_{j=1}^{n} \gamma_{j} x_{j}\right\|^{2} .
$$

Next we prove that $Y$ has type 2 with $T_{2}^{\gamma}(Y) \leqslant K \sqrt{2}$. By an observation in [5] we have

$$
T_{2}^{\gamma}(Y)=\sup \left\{\mathbb{E}\left(\left\|\sum_{j=1}^{n} \gamma_{j} y_{j}\right\|^{2}\right)^{\frac{1}{2}}: n \geqslant 1,\left\|y_{1}\right\|=\cdots=\left\|y_{n}\right\|=n^{-\frac{1}{2}}\right\} .
$$

Fix an integer $n \geqslant 1$ and vectors $y_{1}, \ldots, y_{n} \in Y$ of norm 1 . Let $\left(e_{j}\right)_{j=1}^{n}$ be the standard unit basis of $l_{n}^{2}$ and let $\varepsilon>0$ be arbitrary and fixed. Since $\operatorname{dim} X=\infty$, by Dvoretzky's theorem [4] we can find an isomorphism $T$ from $l_{n}^{2}$ onto an $n$ dimensional subspace $X_{0}$ of $X$ such that $\|T\| \leqslant 1+\varepsilon$ and $\left\|T^{-1}\right\|=1$. Let

$$
x_{j}:=T e_{j}, \quad j=1, \ldots, n .
$$

Clearly, $1 \leqslant\left\|x_{j}\right\| \leqslant 1+\varepsilon$ and for all $1 \leqslant j \neq k \leqslant n$ and $a, b \in \mathbb{R}$ we have

$$
\left\|a x_{j}-b x_{k}\right\| \geqslant\left\|T^{-1}\right\|^{-1}\left\|a e_{j}-b e_{k}\right\|=\sqrt{a^{2}+b^{2}} .
$$

Define $\varphi_{j}: X \rightarrow \mathbb{R}$ by

$$
\varphi_{j}(x):=\max \left\{0,1-\sqrt{2}\left\|x-x_{j}\right\|\right\} .
$$

Then $\varphi_{j}$ is Lipschitz continuous with Lipschitz constant $\left\|\varphi_{j}\right\|_{\text {Lip }} \leqslant \sqrt{2}$, we have $\varphi_{j}\left(x_{j}\right)=1$, and $\varphi_{j} \equiv 0$ outside the open 'sector'

$$
V_{j}:=\left\{x \in X: \exists t>0 \text { such that }\left\|t x-x_{j}\right\|<\frac{1}{2} \sqrt{2}\right\} .
$$

Note that $0 \notin V_{j}$. We claim that the sectors $V_{j}$ are disjoint. Indeed, given $x \in V_{j}$ we choose $t>0$ such that $\left\|t x-x_{j}\right\|<\frac{1}{2} \sqrt{2}$. Then for $j \neq k$ and all $s>0$,

$$
\left\|s x-x_{k}\right\| \geqslant\left\|t^{-1} s x_{j}-x_{k}\right\|-\left\|t^{-1} s x_{j}-s x\right\| \stackrel{(*)}{>} \sqrt{t^{-2} s^{2}+1}-\frac{1}{2} t^{-1} s \sqrt{2} \stackrel{(* *)}{\geqslant} \frac{1}{2} \sqrt{2} .
$$

In $(*)$ we used $(3)$ and the choice of $t$, while $(* *)$ follows from the inequality $\sqrt{c^{2}+1}-\frac{1}{2} c \sqrt{2} \geqslant \frac{1}{2} \sqrt{2}$.

Define $\psi_{j}: X \rightarrow \mathbb{R}$ by

$$
\psi_{j}(x):=a_{j} \varphi_{j}\left(a_{j}^{-1} x\right)
$$

where the $a_{1}, \ldots, a_{n}>0$ are chosen as in (ii). Then $\psi_{j}$ is Lipschitz continuous with Lipschitz constant $\left\|\psi_{j}\right\|_{\text {Lip }} \leqslant \sqrt{2}$, we have $\psi_{j}\left(a_{j} x_{j}\right)=a_{j}$, and $\psi_{j} \equiv 0$ outside $V_{j}$. Define $f: X \rightarrow Y$ by

$$
f(x):=\sum_{j=1}^{n} \psi_{j}(x) y_{j}
$$


It is clear that $f(0)=0$ and $f\left(a_{j} x_{j}\right)=a_{j} y_{j}$. We claim that $f \in \operatorname{Lip}_{0}(X, Y)$ with $\|f\|_{\text {Lip }} \leqslant \sqrt{2}$. If $x, x^{\prime} \in V_{j}$ for some $j$, then by the disjointness of $V_{j}$ with the other $V_{k}$ 's and the fact that $\left\|y_{j}\right\|=1$ we obtain

$$
\left\|f(x)-f\left(x^{\prime}\right)\right\|=\left\|y_{j}\right\|\left|\psi_{j}(x)-\psi_{j}\left(x^{\prime}\right)\right| \leqslant \sqrt{2}\left\|x-x^{\prime}\right\| .
$$

If $x \in V_{j}$ and $x^{\prime} \in V_{k}$ for $j \neq k$, we choose convex combinations $\xi$ and $\xi^{\prime}$ of $x$ and $x^{\prime}$, say $\xi=(1-s) x+s x^{\prime}$ and $\xi^{\prime}=(1-t) x+t x^{\prime}$ with $0 \leqslant s \leqslant t \leqslant 1$, such that $\xi \in \partial V_{j}$ and $\xi^{\prime} \in \partial V_{k}$. Clearly, $f(\xi)=f\left(\xi^{\prime}\right)=0$. It follows from the previous case that

$$
\begin{aligned}
\left\|f(x)-f\left(x^{\prime}\right)\right\| & \leqslant\|f(x)-f(\xi)\|+\left\|f\left(\xi^{\prime}\right)-f\left(x^{\prime}\right)\right\| \\
& \leqslant \sqrt{2}\|x-\xi\|+\sqrt{2}\left\|\xi^{\prime}-x^{\prime}\right\| \\
& =\sqrt{2}(s+(1-t))\left\|x-x^{\prime}\right\| \leqslant \sqrt{2}\left\|x-x^{\prime}\right\| .
\end{aligned}
$$

The case where $x \in V_{j}$ and $x^{\prime} \notin \bigcup_{k} V_{k}$ is handled similarly. Finally if $x, x^{\prime} \notin \bigcup_{k} V_{k}$, then $f(x)=f\left(x^{\prime}\right)=0$. This concludes the proof of the claim.

Recalling that $f(0)=0,\|f\|_{\text {Lip }} \leqslant \sqrt{2},\|T\| \leqslant 1+\varepsilon,\left\|y_{j}\right\|=1$, we obtain

$$
\begin{aligned}
\mathbb{E}\left\|\sum_{j=1}^{n} \gamma_{j} y_{j}\right\|^{2} & =\mathbb{E}\left\|\sum_{j=1}^{n} \gamma_{j} a_{j}^{-1} f\left(a_{j} x_{j}\right)\right\|^{2} \leqslant 2 K^{2} \mathbb{E}\left\|\sum_{j=1}^{n} \gamma_{j} x_{j}\right\|^{2} \\
& \leqslant 2 K^{2}(1+\varepsilon)^{2} \mathbb{E}\left\|\sum_{j=1}^{n} \gamma_{j} e_{j}\right\|^{2}=2 K^{2}(1+\varepsilon)^{2} \sum_{j=1}^{n}\left\|y_{j}\right\|^{2} .
\end{aligned}
$$

By (1) this proves that $Y$ has type 2 with $T_{2}^{\gamma}(Y) \leqslant K \sqrt{2}(1+\varepsilon)$. Since $\varepsilon>0$ was arbitrary, the proof is complete.

(iii) $\Rightarrow$ (i): Assume that $X$ has cotype 2 and $Y$ has type 2 . For all $x_{1}, \ldots, x_{n} \in X$, $a_{1}, \ldots, a_{n}>0$, and $f_{1}, \ldots, f_{n} \in \operatorname{Lip}_{0}(X, Y)$ we have

$$
\begin{aligned}
\mathbb{E}\left\|\sum_{j=1}^{n} \gamma_{j} a_{j}^{-1} f_{j}\left(a_{j} x_{j}\right)\right\|^{2} & \leqslant T_{2}^{\gamma}(Y)^{2}\left(\max _{1 \leqslant j \leqslant n}\left\|f_{j}\right\|_{\text {Lip }}\right)^{2} \sum_{j=1}^{n} a_{j}^{-2}\left\|a_{j} x_{j}\right\|^{2} \\
& \leqslant T_{2}^{\gamma}(Y)^{2}\left(\max _{1 \leqslant j \leqslant n}\left\|f_{j}\right\|_{\text {Lip }}\right)^{2} C_{2}^{\gamma}(X)^{2} \mathbb{E}\left\|\sum_{j=1}^{n} \gamma_{j} x_{j}\right\|^{2} .
\end{aligned}
$$

By a celebrated theorem of Kwapien [8], a Banach space $X$ has type 2 and cotype 2 if and only if $X$ is isomorphic to a Hilbert space. Thus if we take $X=Y$ in the theorem, then assertion (iii) may be replaced by:

(iii) $^{\prime} X$ is isomorphic to a Hilbert space.

In Theorem 1 we may replace the Gaussian sequence $\left(\gamma_{j}\right)_{j \geqslant 1}$ by a Rademacher sequence $\left(r_{j}\right)_{j \geqslant 1}$, in which case we obtain the estimates

$$
C_{2}^{r}(X) \leqslant K \quad \text { and } \quad T_{2}^{r}(Y) \leqslant \frac{2}{\sqrt{\pi}} K
$$

Here $C_{2}^{r}(X)$ and $T_{2}^{r}(Y)$ denote the Rademacher cotype 2 constant of $X$ and the Rademacher type 2 constant of $Y$, respectively. For the second estimate we recall 
from $\left[9\right.$, Lemma 4.5] that $T_{2}^{r}(X) \leqslant \frac{1}{m_{1}^{\gamma}} T_{2}^{\gamma}(X)$, where $m_{1}^{\gamma}:=\mathbb{E}\left|\gamma_{j}\right|=\sqrt{2 / \pi}$ and that by an observation in [5] we have

$$
T_{2}^{\gamma}(Y)=\sup \left\{\mathbb{E}\left(\left\|\sum_{j=1}^{n} r_{j} y_{j}\right\|^{2}\right)^{\frac{1}{2}}: n \geqslant 1,\left\|y_{1}\right\|=\cdots=\left\|y_{n}\right\|=n^{-\frac{1}{2}}\right\} .
$$

The proof of (ii) $\Rightarrow$ (iii) may now be repeated verbatim.

Next let $\left(\xi_{j}\right)_{j \geqslant 1}$ be an arbitrary sequence of i.i.d. symmetric random variables with $\mathbb{E}\left|\xi_{j}\right|^{2}=1$. We denote by $T_{2}^{\xi}(X)$ and $C_{2}^{\xi}(X)$ the $\xi$-type 2 and $\xi$-cotype 2 constant of a Banach space, respectively. By a standard randomization argument, every Banach space $X$ with (co)type 2 has $\xi$-(co)type 2 with constants $T_{2}^{\xi}(X) \leqslant$ $T_{2}^{r}(X)$ and $C_{2}^{\xi}(X) \leqslant C_{2}^{r}(X)$. Conversely, if $X$ has $\xi$-type 2, then again by $[9$, Lemma 4.5],

$$
\left(\mathbb{E}\left\|\sum_{j=1}^{n} r_{j} x_{j}\right\|^{2}\right)^{\frac{1}{2}} \leqslant \frac{1}{m_{1}^{\xi}}\left(\mathbb{E}\left\|\sum_{j=1}^{n} \xi_{j} x_{j}\right\|^{2}\right)^{\frac{1}{2}} \leqslant \frac{1}{m_{1}^{\xi}} T_{2}^{\xi}(X)\left(\sum_{j=1}^{n}\left\|x_{j}\right\|^{2}\right)^{\frac{1}{2}},
$$

where $m_{1}^{\xi}:=\mathbb{E}\left|\xi_{j}\right|$. It follows that $X$ has type 2 with $T_{2}^{r}(X) \leqslant \frac{1}{m_{1}^{\xi}} T_{2}^{\xi}(X)$. If $X$ has $\xi$-cotype 2 and all moments of $\xi_{j}$ are finite, then $X$ has finite cotype (we are grateful to Tuomas Hytönen for pointing this out to us). In fact, by means of elementary estimates it can be shown that $c_{0}$ does not have finite $\xi$-cotype. The Rademacher cotype 2 of $X$ then follows from the Maurey-Pisier theorem; cf. [9, Section 9.2].

At the expense of slightly worse estimate for the type 2 constant it is possible to generalize Theorem 1 to sequences of random variables $\left(\xi_{j}\right)_{j \geqslant 1}$ as above. This is achieved by a slightly modified argument which does not require normalizations as in (1) and (4) and which has the additional virtue that for each $n$ the scalars $a_{1}, \ldots, a_{n}$ are allowed to depend not only on the vectors $x_{1}, \ldots, x_{n}$ but also on the function $f$.

Theorem 2. Let $X$ and $Y$ be Banach spaces with $\operatorname{dim} X=\infty$ and $\operatorname{dim} Y \geqslant 1$, and let $\xi=\left(\xi_{j}\right)_{j \geqslant 1}$ be a sequence of i.i.d. random variables with $\mathbb{E}\left|\xi_{j}\right|^{2}=1$. The following assertions are equivalent:

(i) For all $f_{1}, \ldots, f_{n} \in \operatorname{Lip}_{0}(X, Y)$, all finite sequences $x_{1}, \ldots, x_{n} \in X$, and all scalars $a_{1}, \ldots, a_{n}>0$ we have

$$
\left(\mathbb{E}\left\|\sum_{j=1}^{n} \xi_{j} a_{j}^{-1} f_{j}\left(a_{j} x_{j}\right)\right\|^{2}\right)^{\frac{1}{2}} \leqslant K\left(\max _{1 \leqslant j \leqslant n}\left\|f_{j}\right\|_{\text {Lip }}\right)\left(\mathbb{E}\left\|\sum_{j=1}^{n} \xi_{j} x_{j}\right\|^{2}\right)^{\frac{1}{2}}
$$

where $K$ is a constant depending on $X$ and $Y$ only.

(ii) For all $f \in \operatorname{Lip}_{0}(X, Y)$ and all finite sequences $x_{1}, \ldots, x_{n} \in X$ there exist scalars $a_{1}, \ldots, a_{n}>0$ such that

$$
\left(\mathbb{E}\left\|\sum_{j=1}^{n} \xi_{j} a_{j}^{-1} f\left(a_{j} x_{j}\right)\right\|^{2}\right)^{\frac{1}{2}} \leqslant K\|f\|_{\text {Lip }}\left(\mathbb{E}\left\|\sum_{j=1}^{n} \xi_{j} x_{j}\right\|^{2}\right)^{\frac{1}{2}},
$$

where $K$ is a constant depending on $X$ and $Y$ only.

(iii) $X$ has $\xi$-cotype 2 and $Y$ has $\xi$-type 2 .

If (ii) holds, then $C_{2}^{\xi}(X) \leqslant K$ and $T_{2}^{\xi}(Y) \leqslant(1+2 \sqrt{2}) K$. If the $\xi_{j}$ have finite moments of all orders, then (iii) is equivalent to

(iv) $X$ has cotype 2 and $Y$ has type 2. 
Proof. Only the proof that $Y$ has $\xi$-type 2 in the implication (ii) $\Rightarrow$ (iii) needs to be adapted. Fix arbitrary nonzero vectors $y_{1}, \ldots, y_{n} \in Y$. Following the arguments in the proof of (ii) $\Rightarrow$ (iii) in Theorem 1, we replace (2) by

$$
x_{j}:=\left\|y_{j}\right\| T e_{j}, \quad j=1, \ldots, n,
$$

and define $\varphi_{j}: X \rightarrow \mathbb{R}$ by $\varphi(0)=0$ and

$$
\varphi_{j}(x):=\max \left\{0,1-\sqrt{2}(1+\varepsilon) d_{j}(x)\right\}\|x\|,
$$

where $d_{j}: X \backslash\{0\} \rightarrow \mathbb{R}$ is the function

$$
d_{j}(x):=\left\|\frac{x}{\|x\|}-\frac{x_{j}}{\left\|x_{j}\right\|}\right\|
$$

Then $\varphi_{j}$ is Lipschitz continuous with $\left\|\varphi_{j}\right\|_{\text {Lip }} \leqslant L_{\varepsilon}:=2 \sqrt{2}(1+\varepsilon)+1$, we have $\varphi_{j}\left(a x_{j}\right)=a\left\|x_{j}\right\|$ for all $a>0$, and $\varphi_{j} \equiv 0$ outside the sector

$$
V_{j}:=\left\{x \in X \backslash\{0\}: d_{j}(t x)<\frac{1}{2} \sqrt{2}(1+\varepsilon)^{-1}\right\} .
$$

As before, $V_{j}$ and $V_{k}$ are disjoint for $j \neq k$. Indeed if $x \in V_{j}$, then for $j \neq k$ we have

$$
\begin{aligned}
\left\|\frac{x}{\|x\|}-\frac{x_{k}}{\left\|x_{k}\right\|}\right\| & \geqslant\left\|\frac{x_{j}}{\left\|x_{j}\right\|}-\frac{x_{k}}{\left\|x_{k}\right\|}\right\|-\left\|\frac{x_{j}}{\left\|x_{j}\right\|}-\frac{x}{\|x\|}\right\| \\
& >\sqrt{\left\|T e_{j}\right\|^{-2}+\left\|T e_{k}\right\|^{-2}}-\frac{1}{2} \sqrt{2}(1+\varepsilon)^{-1} \\
& \geqslant \sqrt{2}(1+\varepsilon)^{-1}-\frac{1}{2} \sqrt{2}(1+\varepsilon)^{-1}=\frac{1}{2} \sqrt{2}(1+\varepsilon)^{-1},
\end{aligned}
$$

which shows that $x \notin S_{k}$. Define $f: X \rightarrow Y$ by

$$
f(x)=\sum_{j=1}^{n} \varphi_{j}(x) \frac{y_{j}}{\left\|x_{j}\right\|} .
$$

Then $f(0)=0, f\left(a x_{j}\right)=a y_{j}=a f\left(x_{j}\right)$ for $a>0$, and $f$ is Lipschitz continuous with $\|f\|_{\text {Lip }} \leqslant L_{\varepsilon}$. With the $a_{1}, \ldots, a_{n}>0$ as in (ii), estimating as before we obtain

$$
\mathbb{E}\left\|\sum_{j=1}^{n} \xi_{j} y_{j}\right\|^{2}=\mathbb{E}\left\|\sum_{j=1}^{n} \xi_{j} a_{j}^{-1} f\left(a_{j} x_{j}\right)\right\|^{2} \leqslant\|f\|_{\text {Lip }}^{2} K^{2}(1+\varepsilon)^{2} \sum_{j=1}^{n}\left\|y_{j}\right\|^{2} .
$$

This proves that $Y$ has $\xi$-type 2 with

$$
T_{2}^{\xi}(Y) \leqslant K\|f\|_{\text {Lip }}(1+\varepsilon) \leqslant K(1+2 \sqrt{2}(1+\varepsilon))(1+\varepsilon) .
$$

Since $\varepsilon>0$ was arbitrary, the proof is complete.

If the $\xi_{j}$ have finite moments of all orders, for $X=Y$ we obtain an isomorphic characterization of Hilbert spaces as before.

Theorems 1 and 2 bear a striking resemblance to [1, Proposition 1.13] which states that $X$ has type 2 and $Y$ has cotype 2 if and only if every uniformly bounded family $\mathscr{T}$ in $\mathscr{L}(X, Y)$ is $R$-bounded. Recall that $\mathscr{T}$ is called $R$-bounded if there exists a constant $K$ such that for all choices $x_{1}, \ldots, x_{n} \in X$ we have

$$
\left(\mathbb{E}\left\|\sum_{j=1}^{n} r_{j} T_{j} x_{j}\right\|^{2}\right)^{\frac{1}{2}} \leqslant K\left(\mathbb{E}\left\|\sum_{j=1}^{n} r_{j} x_{j}\right\|^{2}\right)^{\frac{1}{2}} .
$$

This result is elementary (it suffices to consider suitably chosen families of rank one operators) and the role of the Rademacher variables can be replaced by any 
i.i.d. sequence of mean zero random variables with finite second moment. The precise relationship between [1, Proposition 1.13] and our results remains unclear, since we see no obvious way to relate finitely many linear operators in $\mathscr{L}(X, Y)$ to a single nonlinear function in $\operatorname{Lip}_{0}(X, Y)$. In this connection it is worthwhile to point out that it appears to be an unsolved open problem whether for every pair of Banach spaces $X$ and $Y$ there exists a constant $c(X, Y)$ such that, given any distinct elements $x_{1}, \ldots, x_{n} \in X$ and elements $y_{1}, \ldots, y_{n} \in Y$, there exists a Lipschitz function $f: X \rightarrow Y$ satisfying $f\left(x_{j}\right)=y_{j}$ for all $j=1, \ldots, n$ and

$$
\|f\|_{\text {Lip }} \leqslant c(X, Y) \max _{\substack{1 \leqslant j, k \leqslant n \\ j \neq k}} \frac{\left\|y_{j}-y_{k}\right\|}{\left\|x_{j}-x_{k}\right\|} .
$$

The important point here is that $c(X, Y)$ should be independent of $n$. Indeed, it was shown in [6] that for fixed $n,(5)$ can be achieved with a constant $c(n, X, Y)$ of order $\log n$.

As an application of Theorem 1 we will prove next that $\operatorname{Lip}_{0}(X)$ acts in the operator ideal $\gamma\left(l^{2}, X\right)$ of $\gamma$-radonifying operators from $l^{2}$ to $X$ if and only if $X$ is isomorphic to a Hilbert space.

Let $H$ be a Hilbert space. We denote by $\gamma(H, X)$ the completion of the vector space of all finite rank operators $u: H \rightarrow X$ with respect to the norm

$$
\|u\|_{\gamma(H, X)}:=\sup \left(\mathbb{E}\left\|\sum_{j} \gamma_{j} u h_{j}\right\|^{2}\right)^{\frac{1}{2}} \text {. }
$$

The supremum is taken over all finite orthonormal systems $\left(h_{j}\right)$ in $H$. As is well known, $\gamma(H, X)$ is an operator ideal in the sense that for all bounded linear operators $v: \tilde{H} \rightarrow H$ and $w: X \rightarrow \tilde{X}$ we have $w u v \in \gamma(\tilde{H}, \tilde{X})$ and

$$
\|w u v\|_{\gamma(\tilde{H}, \tilde{X})} \leqslant\|w\|\|u\|_{\gamma(H, X)}\|v\| .
$$

For more information we refer to [3, Chapter 12].

We will be interested in the particular case where $H$ equals $L^{2}:=L^{2}(S, \Sigma, \mu)$ for some $\sigma$-finite measure space $(S, \Sigma, \mu)$ and $u_{\phi}: L^{2} \rightarrow X$ is an integral operator of the form

$$
u_{\phi} h=\int_{S} h(s) \phi(s) d \mu(s), \quad h \in L^{2},
$$

for suitable functions $\phi: S \rightarrow X$. Operators in $\gamma\left(L^{2}, X\right)$ arising in this way have been investigated recently in [7]. If $\phi$ is a simple function, i.e., a function of the form $\sum_{j=1}^{n} \mathbf{1}_{S_{j}} \otimes x_{j}$ with vectors $x_{j}$ taken from $X$ and disjoint sets $S_{j} \in \Sigma$ satisfying $0<\mu\left(S_{j}\right)<\infty$, it is easily checked that $u_{\phi} \in \gamma\left(L^{2}, X\right)$ and by considering the orthonormal functions $h_{j}:=\mu\left(S_{j}\right)^{-\frac{1}{2}} \mathbf{1}_{S_{j}}$, the $\gamma$-norm of $\phi$ is computed as

$$
\left\|u_{\phi}\right\|_{\gamma\left(L^{2}, X\right)}^{2}=\mathbb{E}\left\|\sum_{j=1}^{n} \gamma_{j} u_{\phi} h_{j}\right\|^{2}=\mathbb{E}\left\|\sum_{j=1}^{n} \gamma_{j} \mu\left(S_{j}\right)^{\frac{1}{2}} x_{j}\right\|^{2} .
$$

The subspace of all $u \in \gamma\left(L^{2}, X\right)$ of the form $u=u_{\phi}$ for some simple function $\phi: S \rightarrow X$ will be denoted by $\gamma_{\text {simple }}\left(L^{2}, X\right)$. An easy approximation argument shows that this is a dense subspace of $\gamma\left(L^{2}, X\right)$.

If $X$ has type 2, the mapping $\phi \mapsto u_{\phi}$ defined for simple functions $\phi$ as above, extends to a continuous embedding from $L^{2}(X):=L^{2}(S, \Sigma, \mu ; X)$ into $\gamma\left(L^{2}, X\right)$. 
Indeed, for a simple function $\phi=\sum_{j=1}^{n} \mathbf{1}_{S_{j}} \otimes x_{j}$ we have, using (7),

$$
\begin{aligned}
\left\|u_{\phi}\right\|_{\gamma\left(L^{2}, X\right)}^{2} & =\mathbb{E}\left\|\sum_{j=1}^{n} \gamma_{j} \mu\left(S_{j}\right)^{\frac{1}{2}} x_{j}\right\|^{2} \\
& \leqslant T_{2}^{\gamma}(X)^{2} \sum_{j=1}^{n} \mu\left(S_{j}\right)\left\|x_{j}\right\|^{2}=T_{2}^{\gamma}(X)^{2}\|\phi\|_{L^{2}(X)}^{2},
\end{aligned}
$$

and the claim follows by a density argument. Similarly, if $X$ has cotype 2, then $u_{\phi} \mapsto \phi$ extends to a continuous embedding from $\gamma\left(L^{2}, X\right)$ into $L^{2}(X)$.

If $\phi=\sum_{j=1}^{n} \mathbf{1}_{S_{j}} \otimes x_{j}$ is a simple $X$-valued function, then for each $f \in \operatorname{Lip}_{0}(X, Y)$,

$$
f(\phi)=\sum_{j=1}^{n} \mathbf{1}_{S_{j}} \otimes f\left(x_{j}\right)
$$

is a simple $Y$-valued function. In this way we obtain a mapping $\tilde{f}: \gamma_{\text {simple }}\left(L^{2}, X\right) \rightarrow$ $\gamma_{\text {simple }}\left(L^{2}, Y\right)$ by putting

$$
\tilde{f}\left(u_{\phi}\right):=u_{f(\phi)}
$$

We are interested in conditions ensuring that $\tilde{f}$ extends to a Lipschitz continuous mapping from $\gamma\left(L^{2}, X\right)$ to $\gamma\left(L^{2}, Y\right)$. From $f(0)=0$ we see that a necessary condition is that there should exist a constant $K$ such that

$$
\left\|u_{f(\phi)}\right\|_{\gamma\left(L^{2}, Y\right)} \leqslant K\|f\|_{\text {Lip }}\left\|u_{\phi}\right\|_{\gamma\left(L^{2}, X\right)}
$$

for all simple functions $\phi: S \rightarrow X$. The next result gives a converse and relates both conditions to the geometry of the spaces $X$ and $Y$.

Theorem 3. Let $X$ and $Y$ be Banach spaces, let $L^{2}:=L^{2}(S, \Sigma, \mu)$ as before, and assume that $\operatorname{dim} X=\infty, \operatorname{dim} Y \geqslant 1$, and $\operatorname{dim} L^{2}=\infty$. Let $\left(\gamma_{j}\right)_{j \geqslant 1}$ be a sequence of independent standard Gaussian random variables. The following assertions are equivalent:

(i) For all $f \in \operatorname{Lip}_{0}(X, Y)$ and all simple functions $\phi: S \rightarrow X$ we have

$$
\left\|u_{f(\phi)}\right\|_{\gamma\left(L^{2}, Y\right)} \leqslant K\|f\|_{\text {Lip }}\left\|u_{\phi}\right\|_{\gamma\left(L^{2}, X\right)}
$$

where $K$ is a constant depending on $X$ and $Y$ only.

(ii) $X$ has cotype 2 and $Y$ has type 2.

If (i) holds, then $C_{2}^{\gamma}(X) \leqslant K$ and $T_{2}^{\gamma}(Y) \leqslant \sqrt{2} K$, and for all $f \in \operatorname{Lip}_{0}(X, Y)$ the mapping $\tilde{f}$ uniquely extends to an element of $\operatorname{Lip}_{0}\left(\gamma\left(L^{2}, X\right), \gamma\left(L^{2}, Y\right)\right)$ satisfying

$$
\|\tilde{f}\|_{\text {Lip }} \leqslant C_{2}^{\gamma}(X) T_{2}^{\gamma}(Y)\|f\|_{\text {Lip }}
$$

Proof. (i) $\Rightarrow$ (ii): Let $x_{1}, \ldots, x_{n} \in X$ be arbitrary. By the $\sigma$-finiteness of $(S, \Sigma, \mu)$ and the assumption that $\operatorname{dim} L^{2}=\infty$ there exist disjoint sets $S_{1}, \ldots, S_{n} \in \Sigma$ satisfying $0<\mu\left(S_{j}\right)<\infty$ for $j=1, \ldots, n$ and define $\phi: S \rightarrow X$ by $\phi:=\sum_{j=1}^{n} h_{j} \otimes x_{j}$, where $h_{j}=\mu\left(S_{j}\right)^{-1 / 2} \mathbf{1}_{S_{j}}$ for all $j$. It follows from (7) that for all $f \in \operatorname{Lip}_{0}(X, Y)$,

$$
\begin{aligned}
& \left(\mathbb{E}\left\|\sum_{j=1}^{n} \gamma_{j} \mu\left(S_{j}\right)^{\frac{1}{2}} f\left(\mu\left(S_{j}\right)^{-\frac{1}{2}} x_{j}\right)\right\|^{2}\right)^{\frac{1}{2}}=\left\|u_{f(\phi)}\right\|_{\gamma\left(L^{2}, Y\right)} \\
& \quad \leqslant K\|f\|_{\text {Lip }}\left\|u_{\phi}\right\|_{\gamma\left(L^{2}, X\right)}=K\|f\|_{\text {Lip }}\left(\mathbb{E}\left\|\sum_{j=1}^{n} \gamma_{j} x_{j}\right\|^{2}\right)^{\frac{1}{2}} .
\end{aligned}
$$


By an application of Theorem 1 with $a_{j}=\mu\left(S_{j}\right)^{-\frac{1}{2}}$ we obtain (ii).

(ii) $\Rightarrow$ (i): Assume that $X$ has cotype 2 and $Y$ has type 2 and fix $f \in \operatorname{Lip}_{0}(X, Y)$. For simple functions $\phi, \psi: S \rightarrow X$ we have, by (8) and its cotype 2 analogue,

$$
\begin{aligned}
& \left\|\tilde{f}\left(u_{\phi}\right)-\tilde{f}\left(u_{\psi}\right)\right\|_{\gamma\left(L^{2}, Y\right)} \\
& \quad=\left\|u_{f(\phi)}-u_{f(\psi)}\right\|_{\gamma\left(L^{2}, Y\right)} \leqslant T_{2}^{\gamma}(Y)\|f(\phi)-f(\psi)\|_{L^{2}(Y)} \\
& \quad \leqslant T_{2}^{\gamma}(Y)\|f\|_{\text {Lip }}\|\phi-\psi\|_{L^{2}(X)} \leqslant C_{2}^{\gamma}(X) T_{2}^{\gamma}(Y)\|f\|_{\text {Lip }}\left\|u_{\phi}-u_{\psi}\right\|_{\gamma\left(L^{2}, X\right)} .
\end{aligned}
$$

Since $\gamma_{\text {simple }}\left(L^{2}, X\right)$ is dense in $\gamma\left(L^{2}, X\right)$ it follows that $\tilde{f}$ has a unique Lipschitz continuous extension from $\gamma\left(L^{2}, X\right)$ to $\gamma\left(L^{2}, Y\right)$ with $\|\tilde{f}\|_{\text {Lip }} \leqslant C_{2}^{\gamma}(X) T_{2}^{\gamma}(Y)\|f\|_{\text {Lip }}$. This proves the final assertion, and (i) follows by taking $\psi=0$.

Theorem 3 is motivated by the result from $[10,11]$ that a function $\phi:(0, T) \rightarrow X$ is stochastically integrable with respect to a Brownian motion if and only if the operator $u_{\phi}$ is well defined and belongs to $\gamma\left(L^{2}(0, T), X\right)$. The question whether $\tilde{f}$ extends continuously to $\gamma\left(L^{2}(0, T), X\right)$ for all $f \in \operatorname{Lip}_{0}(X, X)$ thus amounts to asking whether $f(\phi)$ is stochastically integrable whenever $\phi$ has this property. This question arises naturally in the study of stochastic differential equations in $X$ driven by multiplicative noise satisfying Lipschitz conditions; cf. [2] for the Hilbert space case. Theorem 3 applied to $X=Y$ shows that in general the answer is negative unless $X$ is isomorphic to a Hilbert space.

Acknowledgment - We thank Tuomas Hytönen and the anonymous referee for helpful remarks.

\section{REFERENCES}

[1] W. AREndt And S. Bu, The operator-valued Marcinkiewicz multiplier theorem and maximal regularity, Math. Z. 240 (2002), 311-343.

[2] G. Da Prato and J. ZabczyK, "Stochastic Equations in Infinite Dimensions", Encyclopedia of Mathematics and its Applications, Cambridge University Press, Cambridge, 1992.

[3] J. Diestel, H. Jarchow and A. Tonge, "Absolutely Summing Operators", Cambridge Studies in Adv. Math., Vol. 34, Cambridge, 1995.

[4] A. Dvoretzky, Some results on convex bodies and Banach spaces, 1961 Proc. Internat. Sympos. Linear Spaces (Jerusalem, 1960), 123-160.

[5] R.C. James, Nonreflexive spaces of type 2, Israel J. Math. 30 (1978), 1-13.

[6] W.B. Johnson, J. Lindenstrauss, and G. Schechtman, Extensions of Lipschitz maps into Banach spaces, Israel J. Math. 54 (1986), 129-138.

[7] N. Kalton And L. Weis, The $H^{\infty}$-functional calculus and square function estimates, preprint.

[8] S. KWAPIEŃ, Isomorphic characterizations of inner product spaces by orthogonal series with vector valued coefficients, Studia Math. 44 (1972), 583-595.

[9] M. Ledoux and M. Talagrand, "Probability in Banach Spaces. Isoperimetry and Processes", Ergebnisse der Mathematik und ihrer Grenzgebiete, Vol. 23, Springer-Verlag, Berlin, 1991.

[10] J.M.A.M. van NeERven And L. Weis, Stochastic integration of functions with values in a Banach space, Studia Math. 166 (2005), 131-170.

[11] J. Rosiński And Z. Suchanecki, On the space of vector-valued functions integrable with respect to the white noise, Colloq. Math. 43 (1980), 183-201.

Delft Institute of Applied Mathematics, Technical University of Delft, P.O. Box 5031, 2600 GA Delft, The Netherlands

E-mail address: J.vanNeerven@math.tudelft.nl, M.C.Veraar@math.tudelft.nl 